\title{
Gastrointestinal Stromal Tumors (GIST)
}

\author{
Rahman G. Barry, Thao T. Wolbert and \\ David A. Denning \\ Additional information is available at the end of the chapter
}

http://dx.doi.org/10.5772/intechopen.74290

\begin{abstract}
Gastrointestinal stromal tumors (GISTs) are the most common mesenchymal neoplasms of the gastrointestinal tract, occurring predominantly in the stomach and small intestine. These tumors account for up to $3 \%$ of all gastrointestinal malignancies, with a reported annual incidence of $10-15$ cases per million population. GISTs are thought to originate from interstitial cells of Cajal (ICC) or ICC precursor cells, and are characterized by activating mutations in the KIT (CD117) and platelet-derived growth factor receptor alpha (PDGFR $\alpha$ ) proto-oncogenes in $85-95 \%$ of all cases. The clinical presentation and tumor biology of GISTs are widely variable, with several advances being made over the past two decades in the understanding of GIST tumor biology and pathophysiology. This has led to a paradigm shift in management from the purely surgical approach of the past, to a multi-modality treatment strategy with a greater role for targeted therapies in the form of tyrosine kinase inhibitors, resulting in significantly improved patient outcomes.
\end{abstract}

Keywords: gastrointestinal stromal tumor (GIST), stromal tumors, gastric tumors, KIT mutation, imatinib

\section{Introduction}

Gastrointestinal stromal tumors (GIST) represents the most common mesenchymal tumor of the gastrointestinal (GI) tract, accounting for up to 3\% of all GI malignancies [1, 2]. The history of GIST dates back several decades, with early classifications including GI schwannomas, leiomyomas, leiomyoblastomas and leiomyosarcomas based on the histological findings and variable differentiation associated with these tumors. With advances in immunohistochemical staining techniques and improvements in microscopic structural imaging, GISTs became recognized as a distinct entity. Although the term GIST was originally coined in 1983 by Mazur 
and Clark, it was not until 1998 that Hirota et al. discovered a gain-of-function mutation in the KIT proto-oncogene associated with GISTs $[3,4]$. This finding led to a greater understanding of these tumors, and an eventual reclassification of GI sarcomas.

The reported annual incidence of GIST is 10-15 cases per million population, with over 5000 new cases being diagnosed each year within the United States [5-8]. GISTs can occur at any age, however, more than $80 \%$ have been reported in individuals over 50 , with the median age of presentation at 63 years old $[9,10]$. Most studies have reported no significant gender predilection, with a few studies identifying a slight male predominance $[7,8,11]$. While GISTs can occur anywhere along the GI tract, they are most commonly found in the stomach $(50 \%)$, followed by small intestine $(36 \%)$, colon (7\%), rectum (5\%) and esophagus (1\%) [12-14]. In rare instances, primary GISTs are identified outside of the GI tract, including the omentum, retroperitoneum and mesentery. These account for $<5 \%$ of all GISTs and are termed extragastrointestinal stromal tumors (E-GIST), and could represent metastasis from an undetected primary tumor $[13,15]$.

Immunophenotypically, GISTs have a close resemblance to the interstitial cells of Cajal (ICC), the pacemaker cells of the intestine, suggesting that they either originate from ICC or from ICC precursor cells, both of which express type III tyrosine kinase receptors [14]. Activating mutations in the KIT (CD117) and platelet-derived growth factor receptor alpha (PDGFR $\alpha$ ) proto-oncogenes are responsible for $85-95 \%$ of all GISTs [15]. The vast majority of GISTs occur sporadically, with approximately $5 \%$ occurring in the setting of syndromes, most commonly Neurofibromatosis type 1 [16]. Less frequently associated syndromes include Carney triad and Carney-Stratakis syndrome, as well as an association with desmoid tumors. Tumor biology and behavior occur along a spectrum from benign spindle cell nodules to aggressive sarcomas, however, most GISTs, particularly gastric GISTs, are benign.

\section{Pathobiology}

\subsection{Histopathology}

From a histologic standpoint, GISTs can be divided into three main subtypes based on their microscopic appearance. The most common subtype is spindle cell $(70 \%)$, followed by epithelioid (20\%), and mixed (10\%) subtypes [15]. The spindle cell subtype demonstrates KIT or PDGFR $\alpha$ proto-oncogene mutations in the vast majority of cases, and most commonly affects individuals in their fifth and sixth decades of life $[15,17]$. Due to these molecular expressions, the spindle cell subtype responds well to treatment with Imatinib mesylate (Gleevec $($ )), resulting in a better overall prognosis. The epithelioid subtype is less common, and often seen in a younger, predominantly female population, and more commonly arises from the stomach [15, 18]. In addition, this subtype often lacks KIT and PDGFR $\alpha$ proto-oncogene mutations, and often demonstrated lymphatic metastases, resulting in a poorer prognosis when compared to the spindle cell subtype. It is critical to assess the number of mitoses per 50 high-power fields (HPF) during microscopic analysis as it is an important prognostic indicator, along with tumor size. 


\subsection{Molecular biology}

The identification of the KIT proto-oncogene by Hirota et al. in 1998 led to significant advances in the understanding of GIST molecular pathogenesis and set the stage to allow for breakthroughs in the treatment of this disease. A major revolution in the treatment of GISTs began with the introduction of imatinib mesylate (Gleevec $\left.{ }^{\circledR}\right)$, a tyrosine kinase inhibitor (TKI) with activity against both KIT and PDGFR $\alpha$ proto-oncogene mutations. Before the advent of TKI therapy, GISTs were generally thought to be resistant to drug therapy, due to the ineffectiveness of traditional chemotherapy regimens.

Over the past decade, with more frequent use of imatinib therapy in GISTs, medication resistance in KIT-mutated GISTs is being more frequently encountered. This resistance is classified as either primary or secondary depending on whether they have been on treatment for less than 6 months or greater than 6 months, respectively. Different types of KIT proto-oncogene mutations have been identified, and determination of the specific mutation has been shown to predict the tumor response to tyrosine kinase inhibitor therapy. Most KIT proto-oncogene mutations involve exon 11 (75\%), and portend a better prognosis due to a favorable response to tyrosine kinase inhibitor therapy $[8,19]$. The majority of familial cases of GIST also tend to have exon 11 mutations. Another subset of patients harbor mutations involving exon 9, and is characterized by poor responsiveness to tyrosine kinase inhibition therapy, a shorter progression-free interval, and decreased overall survival. This subset is most frequently seen in non-gastric GISTs, and while traditional imatinib therapy shows limited efficacy, high-dose imatinib or sunitinib malate (Sutent ${ }^{\circledR}$ ), a multiple tyrosine kinase receptor inhibitor, has had significant treatment success [20]. The exon 18 D $842 \mathrm{~V}$ mutation is the most common PDGFR $\alpha$ proto-oncogene mutation, and demonstrates resistance to tyrosine kinase inhibitor therapy, portending a poor prognosis.

Approximately $75-80 \%$ of GISTs harbor mutations in the KIT proto-oncogene, and approximately $10 \%$ of GISTs have a gain-of-function mutation in the PDGFR $\alpha$ tyrosine kinase receptor (Table 1) [21]. While the vast majority of GISTs demonstrate mutations in KIT or PDGFR $\alpha$, the remaining $10-15 \%$ do not have mutations in these exons, and are known as wild-type (WT) genotypes. These WT genotypes are characteristically very resistant to tyrosine kinase inhibition therapy. Almost 10\% of WT GISTs harbor a BRAF V600E mutation on exon 15, and are more commonly seen in small intestine GISTs [22]. Secondary resistance in patients who have been on imatinib therapy for over 6 months commonly occurs due to secondary KIT proto-oncogene mutations involving exon 13 and 14, or from mutations involving other tyrosine kinases [23]. These secondary mutations are more frequently encountered with epithelioid or mixed histology and have a predilection for lymphatic metastasis, portending a poor prognosis [15, 24, 25]. More recent studies on WT GISTs have revealed defects in the Krebs cycle enzyme succinate dehydrogenase (SDH), due to either a mutation or abnormal gene methylation, with WT GISTs now commonly being referred to as SDH-deficient GISTs [26, 27].

Numerous receptors and markers have been found to be associated with GISTs. KIT and PDGFR $\alpha$ mutations occur in up to $95 \%$ of cases, followed by expression of CD34 (60-70\%), smooth muscle actin (ACAT2-30-40\%), S100 (5\%), desmin (DES-1-2\%) and keratin (1-2\%) [28-30]. More recently, gene microarray analyses have identified additional markers for 


\begin{tabular}{|c|c|c|c|}
\hline Genetic Type & Distribution & Imatinib Response & Features \\
\hline \multicolumn{4}{|l|}{ KIT mutations } \\
\hline -Exon 9 & SB/Colon & High-dose imatinib & Biologically aggressive \\
\hline \multirow[t]{3}{*}{-Exon 11} & All sites & Responsive & Longer event-free and overall survival; \\
\hline & & & $\begin{array}{l}\text { Common in Familial GISTs; Deletions associated } \\
\text { with malignant course }\end{array}$ \\
\hline & & & Duplications favorable \\
\hline -Exon 13 & All sites & Responsive & Spindle cell subtype; More common in SB \\
\hline -Exon 17 & All sites & Responsive & Spindle cell subtype; \\
\hline \multicolumn{4}{|c|}{ PDGFR $\alpha$ mutations } \\
\hline -Exon 12 & All sites & Responsive & $\begin{array}{l}\text { Epithelioid subtype; Indolent course; More } \\
\text { common in stomach }\end{array}$ \\
\hline -Exon 14 & Stomach & Responsive & \\
\hline -Exon 18 D842V & Stomach, E-GIST & Resistant & -Exon 18 (non-D842V) occurs in all sites \\
\hline
\end{tabular}

Table 1. Molecular classification of GISTs.

GIST. The most notable of these is the DOG1 (Discovered on GIST-1) gene, which has been identified in $97 \%$ of all GISTs, and expresses a calcium-gated protein known as anoctinin-1 [31]. When used along with KIT, DOG1 has allowed up to $100 \%$ sensitivity in GIST detection and has also been found to be highly specific for GISTs [32].

\subsection{Gross pathology}

GISTs show significant size variation, ranging from millimeters in size to well over $20 \mathrm{~cm}$ [33]. The tumors originate from muscle rather than epithelium, and are often friable. Tumors are typically well-circumscribed with intact overlying mucosa, but may be nodular or ulcerated, and may occasionally have a pseudocapsule. As the tumor increases in size, it may outgrow its blood supply and demonstrate necrosis, cystic degeneration or hemorrhagic foci. It is necessary to clearly delineate tumor size, as it is a critical anatomic prognostic indication, along with the number of mitoses per $50 \mathrm{HPF}[16,27]$. A size greater than $5 \mathrm{~cm}$ and a mitotic index greater than 5 per $50 \mathrm{HPF}$ correlates with aggressive tumor behavior and a poorer prognosis. A risk assessment scheme based on several large series published by Miettinen et al. demonstrates that for gastric GISTs with a mitotic index 5 per $50 \mathrm{HPF}$ or lower, tumor sizes of 2-5, 5-10 and $>10 \mathrm{~cm}$ resulted in metastasis or tumor-related death in 1.9, 3.6 and $10 \%$ of patients, respectively. Similarly, for gastric GISTs with a mitotic index of more than 5 per $50 \mathrm{HPF}$, tumor sizes of 2-5, 5-10 and $>10 \mathrm{~cm}$ resulted in metastasis or tumor-related death in 16,55 and $86 \%$ of patients, respectively [34, 35].

\subsection{Micro-GIST}

As imaging techniques improve and newer modalities such as endoscopic ultrasound (EUS) are utilized more frequently, small asymptomatic GISTs are being identified in the stomach wall, and are often under $1 \mathrm{~cm}$ in size. The term micro-GIST as described by Scherubl et al. 
has been found to be pathobiologically different from GISTs that become clinically significant. They have a very low proliferative rate and have different KIT mutations than those typically seen on larger tumors, with no reported progression of these tumors [36-38].

\section{Diagnosis}

The majority of GIST cases present with vague, non-specific symptoms, with up to $25 \%$ being diagnosed incidentally on imaging studies performed or other abdominal pathologies or trauma [39]. Specific symptoms often depend on the location of the tumor, tumor size, and presence of metastatic disease. The most common symptoms are GI bleeding, vague abdominal discomfort, and an abdominal mass, with other symptoms including abdominal pain, nausea, vomiting anorexia and early satiety. GISTs tend to displace adjacent structures rather than invade them, and because of their submucosal origin, they can reach comparatively larger sizes before becoming symptomatic. GISTs with high mitotic rates tend to quickly outgrow their blood supply, and can develop a necrotic core, increasing the risk of rupture, fistulization, and intraperitoneal hemorrhage. Specific to their location, GISTs can result in dysphagia in esophageal cases, gastric outlet obstruction with gastric cases, obstructive jaundice in tumors of duodenal origin, and can act as a lead point for intussusception and small bowel obstruction [40-42]. A high index of suspicion remains paramount for accurate preoperative diagnosis given the rarity of this condition.

Multiple diagnostic and treatment guidelines have been published on GIST, including the National Comprehensive Cancer Network (NCCN) and the European Society of Medical Oncology (ESMO) guidelines. A GIST is suspected whenever a well-circumscribed mural or extramural, submucosal mass of any size is identified in the distal esophagus, stomach or small intestine, whether through imaging studies, at the time of surgery, during endoscopy, or on pathology analysis. It is critical to keep GIST in the differential diagnosis of any mass found in the alimentary canal from esophagus to rectum. GISTs remain the most common mesenchymal tumors of all sections of the GI tract with the exceptions of the esophagus, colon, and rectum, where leiomyomas are more common.

The imaging study of choice for initial evaluation of a GIST is a computed tomography (CT) scan of the abdomen and pelvis using oral and intravenous contrast enhancement, with arterial and venous phases. GISTs will typically appear as a well-circumscribed, enhancing mass arising from the gastric or intestinal lumen. Large GISTs, which have outgrown their blood supply and have areas of necrosis, may appear as heterogeneously enhancing masses on CT imaging. It should be noted that CT Imaging in the setting of inadequate gastric distension can make it difficult to identify small GISTs. Even in patients with prior endoscopic imaging, a baseline CT scan is still necessary to determine full tumor extent and to evaluate for liver and peritoneal metastatic disease.

Routine magnetic resonance imaging (MRI) is inferior to CT imaging for the initial evaluation of a GIST due to the constant movement of the stomach, small bowel, and surrounding tissues. Results using MRI with diffusion-weighted imaging (DWI), however, may be comparable to PET/CT imaging [43]. MRI is indicated in evaluation of peri-ampullary and rectal 
GISTs, and to further evaluate for metastatic liver disease, as lesions are occasionally isodense to liver parenchyma on CT. Neither MRI nor CT has been shown to reliably predict tumor invasion into surrounding structures.

Positron Emission Tomography combined by CT imaging (PET/CT) plays a role in GIST diagnosis and management, as these tumors tend to show significant metabolic activity, and therefore lend themselves well to functional imaging with PET using ${ }^{18} \mathrm{~F}$-fluorodeoxyglucose [44]. It is often used for assessment of metastatic disease and early metabolic changes, especially in response to tyrosine kinase inhibitor therapy, which may be informative in the setting of neoadjuvant therapy. PET imaging may also be beneficial in detecting small omental metastases, which may not easily be identified on CT scans, as ${ }^{18} \mathrm{~F}$-fluorodeoxyglucose is not taken up to any significant degree by normal small intestine or omentum. Recently, a large percentage of GISTs have been found to express somatostatin receptors, SSTR1 and SSTR2. This has created a role for somatostatin receptor scintigraphy in evaluation of GISTs, similar to its role in GI and pancreatic neuroendocrine tumors [45].

Endoscopy plays a significant role in the evaluation of GISTs, and provides a means of tissue sampling for pathologic analysis. Upper GI endoscopy is used to evaluate tumors in the esophagus, stomach and duodenum, while colonoscopy is used to evaluate rectal and colon masses. For distal duodenal and jejunoileal masses, which are poorly accessible by traditional endoscopic techniques, there is a role for capsule endoscopy and for double-balloon enteroscopy in evaluating these lesions. Endoscopic ultrasound (EUS) has also been used in the evaluation of suspected GISTs. They are visualized as circumscribed hypodense lesions deep to the mucosa. Tumors with irregular borders, size greater than $4 \mathrm{~cm}$, as well as significant echogenic foci and cystic spaces have been found to correlate with malignant behavior [46].

Endoscopy and EUS offer the advantage of being able to perform a tissue biopsy at the time of the procedure. Unfortunately, endoscopic mucosal biopsies are successful in only $20-30 \%$ of cases, even in GISTs which involve the mucosa or the superficial submucosa. EUS-guided fine-needle aspiration cytology, however, has demonstrated more favorable results, with a diagnostic yield of $76 \%$, and EUS-guided core biopsies with a yield of $97 \%$ [47, 48]. While there is a role for percutaneous biopsies, particularly in large tumors, many institutions have demonstrated superior yields from EUS-guided biopsies [49, 50]. It should be noted, however, that if imaging studies are strongly suggestive of a mesenchymal tumor which appears to be resectable, it is usually not necessary to obtain a tissue diagnosis preoperatively. Conversely, if metastatic disease is evident, or if resectability is questionable, a tissue diagnosis is required to plan for immunohistochemical analysis and targeted molecular therapy.

The pathologic diagnosis of GIST is defined by the morphology, ultrastructure and immunohistochemistry findings. These tumors are mesenchymal in origin and have spindle cell, epithelioid cell or mixed histology with variable mitotic activity, which along with tumor size are of prognostic significance. Immunohistologically, up to $95 \%$ of GISTs stain positive for KIT (CD117). Additional staining for markers such as PDGFR $\alpha$, PI3K, CDKN2A and DOG1 can also be performed. In addition to tumor size and mitotic activity, several pathologic and immunohistochemical findings have been shown to affect prognosis and predict recurrence, including Ki67 proliferation index, presence of diffuse mucosal involvement, tumor rupture, tumor location, completeness of resection, aneuploidy, and telomerase expression [42, 51-57]. 


\section{Management}

\subsection{Primary GIST}

Definitive surgical resection in patients with primary, localized GISTs remains the only chance for cure [58]. The surgical approach to GISTs takes into consideration the typical tumor growth patterns and tends to follow the general principles of management of sarcomas. It is uncommon for GISTs to have lymph node spread, and they usually do not demonstrate significant intramural extension. Given the somewhat predictable pattern of growth and the infrequency of lymph node involvement, extensive tumor resection and lymph node dissections can usually be avoided unless there is gross lymphadenopathy present. Surgical management typically involves a wedge resection of the stomach, or a segmental small bowel resection for small bowel GISTs.

The best long-term results were traditionally thought to be achieved after complete resection with microscopically negative margins (R0 resection), typically via resections with $1-2 \mathrm{~cm}$ margins. Although the goal of surgical resection should always be to obtain an R0 resection, recent studies have demonstrated that tumor size and inherent biologic status play a greater role in predicting survival than do microscopic margins. It is now generally accepted that grossly negative margins (R1) do not lead to significant differences in recurrence-free survival(RFS) when compared to R0 resections, irrespective of the use of adjuvant therapy $[11,59]$. Gross resection to negative margins is generally achievable in $85 \%$ of localized, primary GISTs, with cases of adjacent organ involvement surgically approached with en bloc resection. The American College of Surgeons Oncology Group (ACOSOG) Z9000 and Z9001 studies, in addition to demonstrating no difference in RFS between R0 and R1 resections, demonstrated that surgical resection of tumors $\geq 3 \mathrm{~cm}$ was curative in $70 \%$ of patients at longterm follow-up, underscoring the pivotal role of surgical resection with curative intent in medically fit patients. The NCCN guidelines reflect these results, with recommendations to resect all GISTs with a size over $2 \mathrm{~cm}$.

GISTs involving the colon and small intestine tend to be more aggressive, and warrant resection regardless of size, whereas for gastric GISTs smaller than $2 \mathrm{~cm}$, endoscopic surveillance or serial imaging seems to be a reasonable option. A 2011 study by Kim et al. looking retrospectively at 989 patients with small subepithelial gastric tumors less than $3 \mathrm{~cm}$ in size demonstrated that only $8.5 \%$ of tumors had significant growth at median follow-up of 24 months. Twenty-five patients underwent tumor resection due to concerning growth or finding on imaging, of which 19 were GISTs, and only 3 of these were considered high-risk, with 4 classified as intermediate-risk [60]. The high incidence of small gastric GISTs is reflected in autopsy series where the incidence of subcentimeter gastric GISTs was as high as $22.5 \%$ in persons over 50 years old in a German study, and the incidence of microscopic GISTs was as high as $35 \%$ in gastric resection specimens for gastric cancer in a Japanese study [61, 62].

Gross resection margins of $2-3 \mathrm{~cm}$ for small bowel GISTs, and a microscopically free margin of only several millimeters in gastric GIST wedge resections is generally considered adequate. Greater gastric resections, including partial or total gastrectomy, are usually reserved for larger GISTs, or those which preclude wedge resection based on location [63]. 
Rectal GISTs remain uncommon, however consideration may be given to endoscopic resection based on size, extent, and anatomic relationships. While not common practice, laparoscopic resection of small GISTs, particularly those which are gastric in origin, has been described with good outcomes [64, 65].

Incomplete (R2) resections of GISTs are generally reserved for surgery with palliative intent to alleviate symptoms related to pain, bleeding, or mass effect. Despite the high rate of successful complete gross tumor resection, there has been a historically high recurrence rate of over 50\%, with an associated 5-year mortality rate of approximately $50 \%$ [11, 42 , 53]. The median time to recurrence after an adequate $\mathrm{R} 0$ resection has been found to be between 19 and 25 months, with recurrences typically involving the liver and peritoneum, and is usually not amenable to a repeat resection [11, 53, 66]. GISTs are usually very vascular with a fragile pseudocapsule. Careful intraoperative handling of the tumor is therefore essential to minimize the risk of tumor rupture, which often results in peritoneal recurrence [53].

Open resection of gastric and small intestine GISTs has been the traditional approach to operative management, however, minimally invasive approaches are now being utilized with good outcomes. Laparoscopic resection of gastric and some small intestine GISTs is being attempted with increased frequency, and allows for many of the benefits of laparoscopic surgery, including decreased postoperative pain, a shorter hospital length-of-stay, faster return to work and functional independence, and a better cosmetic outcome [67-71]. In addition to open surgical options for colon and rectal GISTs, endoscopic resection in cases of small rectal GISTs may also be considered. More recently, laparoscopic and endoscopic cooperative surgery (LECS) has been employed in resection of gastric GISTs, where the mucosal dissection of tumors that protrude intraluminally is performed endoscopically [72].

\subsection{Advanced GIST}

Despite adequate surgical resection of localized GISTs, not all operations are curative. Many studies have reported high recurrence rates, up to $50 \%$, after complete tumor resection [11, $40]$. The initial sites of recurrence are most frequently the liver $(50 \%)$, peritoneum $(50 \%)$, or both $(20 \%)$, with bone and lung metastases often occurring later [73]. Both liver and peritoneal involvement in this setting tend to be diffuse and multifocal, with surgery playing a very limited role in the face of recurrent or metastatic disease. Historically, conventional chemotherapy and radiotherapy regimens have been ineffective in treating advanced GISTs [74]. The advent of targeted molecular therapy has revolutionized the treatment of GISTs, with tyrosine kinase inhibitors such as imatinib playing a pivotal role in management.

The clinical value of imatinib for high-risk patients and those with advanced disease was established in the ACOSOG Z9000 and Z9001 trials. The ACOSOG Z9000 trial demonstrated a remarkable improvement in the survival rate of high-risk patients (tumor size $\geq 10 \mathrm{~cm}$, tumor rupture or $<5$ peritoneal metastases) who had undergone complete surgical resection of KITpositive GISTs [75]. The ACOSOG Z9001 trial was a randomized trial comparing the use of imatinib to a placebo in moderate risk GISTs (tumor size $\geq 3 \mathrm{~cm}$ ). There was a significant increase in RFS in the imatinib group, though overall survival was no different in the short-term [76]. 


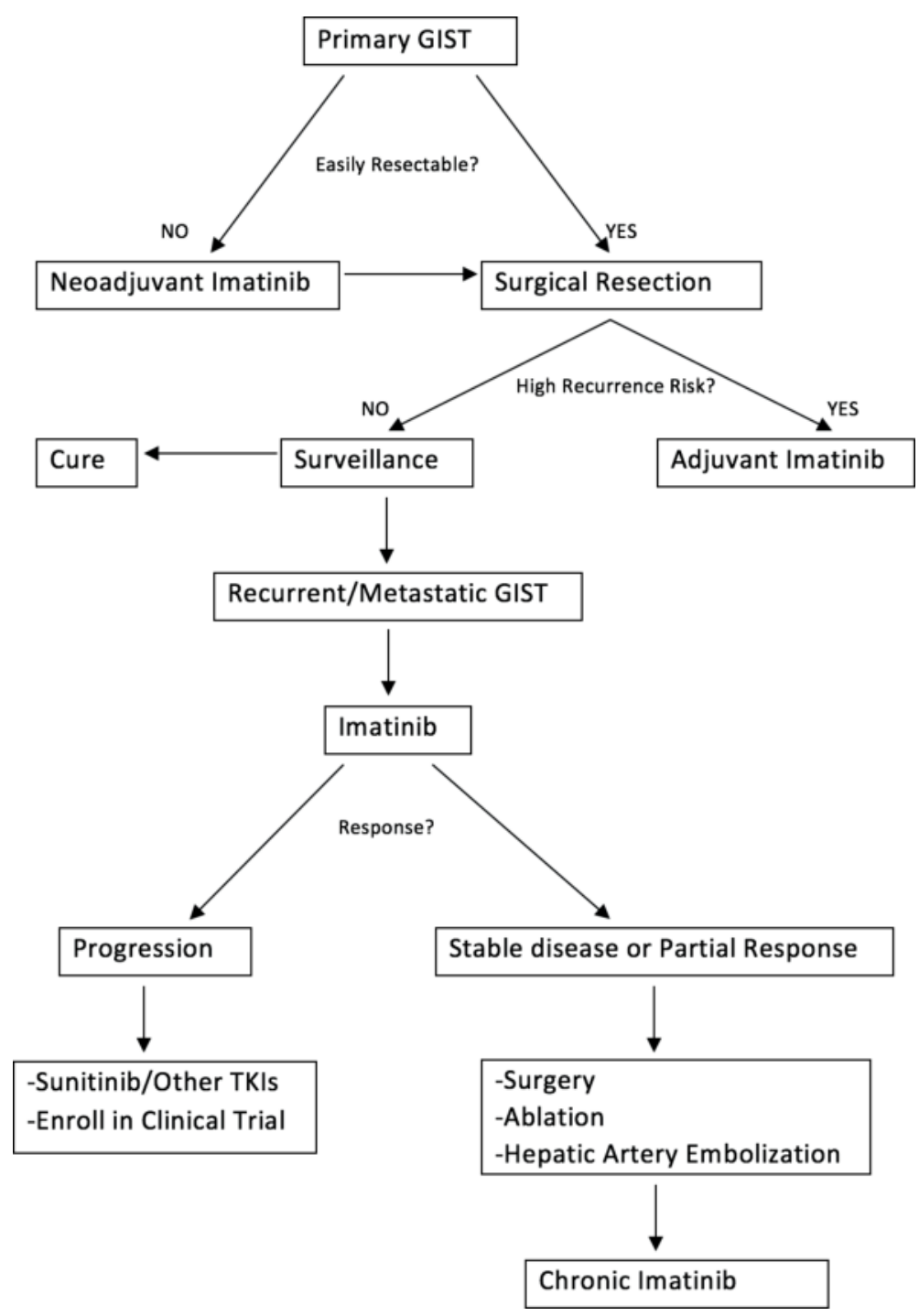

Figure 1. A schematic overview of the approach to management of gastrointestinal stromal Tumors (GISTs). (Adopted from [40]).

Patients receiving imatinib therapy show changes in metabolic activity on PET scans within hours, however, a decrease in tumor size often takes several weeks to months, and tumors may even increase in size initially. For these reasons, the traditional World Health Organization (WHO) and Response Evaluation Criteria in Solid Tumors (RECIST) criteria are not ideal in evaluating response to imatinib therapy in GIST. This response to imatinib therapy is better evaluated by contrast-enhanced CT scans using the Choi criteria, which evaluates for both a decrease in tumor size as well as the tumor density [77, 78]. The use of PET scans have also 
been shown to be very sensitive in evaluating tumor response, given the early changes in tumor metabolic activity [79]. The tumor response to imatinib therapy at day 8 on PET scans have been shown to correlate with 1-year prognosis in unresectable GISTs [79, 80]. Imatinib use in the neoadjuvant setting has not been studied as extensively as adjuvant therapy, but remains very useful in locally advanced or borderline resectable tumors, and tumors in locations where tumor downsizing may allow for a less morbid operation.

Resistance to imatinib therapy unfortunately occurs, and may be of two types as mentioned earlier. Primary resistance occurs in patients who either never had an initial response, or who show disease progression within 6 months of initiating therapy, while secondary resistance occurs in those who show disease progression after 6 months of therapy. This latter group often acquires mutations in KIT or PDGFR $\alpha$ that interfere with imatinib activity [81, 82]. Sunitinib and regorafenib are both newer FDA approved agents which block multiple tyrosine kinase receptors for use in GIST patients with disease progression or intolerance to imatinib. Several other tyrosine kinase inhibitors, including nilotinib, masatinib, dasatinib and sorafenib have been used in cases of resistance to imatinib and sunitinib or in patients with intolerance, however, their role in the management of GISTs continues to be investigated. A schematic overview of the approach to management of GISTs is shown in Figure 1 [40].

\section{Surveillance}

Long-term follow-up is necessary in all patients with GISTs, due to their unpredictable biological behavior. Most recurrences tend to occur within the first 3-5 years, and surveillance recommendations take this into account. Recommendations by the NCCN include performing a history, physical examination, and a contrast-enhanced CT scan of the abdomen and pelvis every 3-6 months for 3-5 years, and then annually thereafter [8]. Recommendations from the European Society of Medical Oncology (ESMO) are based on tumor size and mitotic activity [83]. High-risk tumors (size $\geq 5 \mathrm{~cm}$ and mitoses $\geq 5 / 50 \mathrm{HPF}$ ) require a CT scan of the abdomen and pelvis every 3-4 months for 3 years then every 6 months for 2 years thereafter, while tumors $<5 \mathrm{~cm}$ in size with a mitotic rate of $<5 / 50 \mathrm{HPF}$ should have a CT scan of the abdomen and pelvis every 6 months for 5 years.

\section{Conflict of interest}

The authors have no conflicts of interest to disclose.

\section{Author details}

Rahman G. Barry*, Thao T. Wolbert and David A. Denning

*Address all correspondence to: rahmanbarry@gmail.com

Department of Surgery, Marshall University, Huntington, WV, USA 


\section{References}

[1] Antonescu C. Gastrointestinal stromal tumors. Current Topics in Microbiology and Immunology. 2012;355:41-57

[2] Keung EZ, Raut CP. Management of Gastrointestinal Stromal Tumors. The Surgical Clinics of North America. 2017;97(2):437-452

[3] Mazur MT, Clark HB. Gastric stromal tumors: Reappraisal of histogenesis. The American Journal of Surgical Pathology. 1983;7:507-519

[4] Hirota S, Isozaki K, Moriyama Y, et al. Gain-of-function mutations of c-kit in human gastrointestinal stromal tumors. Science. 1998;279:577-580

[5] Ho MY, Blanke CD. Gastrointestinal stromal tumors: Disease and treatment update. Gastroenterology. 2011;140(5):1372-1376

[6] Quek R, George S. Gastrointestinal stromal tumor: A clinical overview. Hematology/ Oncology Clinics of North America. 2009;23(1):69-78

[7] Raut CP. Gastrointestinal stromal tumors. In: Zinner MJ, Ashley SW, editors. Maingot's Abdominal Operations. 12th ed. New York: McGraw-Hill; 2013. pp. 493-505

[8] Demetri GD, von Mehren M, Antonescu CR, et al. NCCN task force report: Update on the management of patients with gastrointestinal stromal tumors. Journal of the National Comprehensive Cancer Network. 2010;8(Suppl 2):S1-41

[9] Ducimetière F, Lurkin A, Ranchère-Vince D, et al. Incidence of sarcoma histotypes and molecular subtypes in a prospective epidemiological study with central pathology review and molecular testing. PLoS One. 2011;6:e20294

[10] Joensuu H, Vehtari A, Riihimki J, et al. Risk of recurrence of gastrointestinal stromal tumour after surgery: An analysis of pooled population-based cohorts. The Lancet Oncology. 2012;13:265-274

[11] DeMatteo RP, Lewis JJ, Leung D, et al. Two hundred gastrointestinal stromal tumors: Recurrence patterns and prognostic factors for survival. Annals of Surgery. 2000;231(1): $51-58$

[12] Tran T, Davila JA, El-Serag HB. The epidemiology of malignant gastrointestinal stromal tumors: An analysis of 1,458 cases from 1992 to 2000. The American Journal of Gastroenterology. 2005;100(1):162-168

[13] Kim KH, Nelson SD, Kim DH, et al. Diagnostic relevance of overexpressions of PKC- $\theta$ and DOG-1 and KIT/PDGFRA gene mutations in extragastrointestinal stromal tumors: A Korean six-centers study of 28 cases. Anticancer Research. 2012;32:923-937

[14] Miettinen M, Lasota J. Histopathology of gastrointestinal stromal tumor. Journal of Surgical Oncology. 2011;104:865-873

[15] Liegl-Atzwanger B, Fletcher JA, Fletcher CD. Gastrointestinal stromal tumors. Virchows Archiv. 2010;456:111-127 
[16] Miettinen M, Lasota J. Gastrointestinal stromal tumors: Review on morphology, molecular pathology, prognosis, and differential diagnosis. Archives of Pathology \& Laboratory Medicine. 2006;130:1466-1478

[17] Miettinen M, Sobin LH, Lasota J. Gastrointestinal stromal tumors of the stomach: A clinicopathologic, immunohistochemical, and molecular genetic study of 1765 cases with long-term follow-up. The American Journal of Surgical Pathology. 2005;29:52-68

[18] Burkill GJ, Badran M, Al-Muderis O, et al. Malignant gastrointestinal stromal tumor: Distribution, imaging features, and pattern of metastatic spread. Radiology. 2003;226:527-532

[19] Heinrich MC, Owzar K, Corless CL, et al. Correlation of kinase genotype and clinical outcome in the north american intergroup phase iii trial of imatinib mesylate for treatment of advanced gastrointestinal stromal tumor: Calgb 150105 study by cancer and leukemia group b and southwest oncology group. Journal of Clinical Oncology. 2008;26:5360-5367

[20] Debiec-Rychter M, Sciot R, Le Cesne A, et al. Kit mutations and dose selection for imatinib in patients with advanced gastrointestinal stromal tumours. European Journal of Cancer. 2006;42:1093-1103

[21] BarnettCM, Corless CL,Heinrich MC. Gastrointestinal stromal tumors. Molecular markers and genetic subtypes. Hematology/Oncology Clinics of North America. 2013;27:871-888. DOI: 10.1016/j.hoc.2013.07.003

[22] Agaram NP, Wong GC, Guo T, et al. Novel v600e braf mutations in imatinib-naive and imatinib-resistant gastrointestinal stromal tumors. Genes, Chromosomes \& Cancer. 2008;47:853-859

[23] Maleddu A, Pantaleo MA, Nannini M, et al. Mechanisms of secondary resistance to tyrosine kinase inhibitors in gastrointestinal stromal tumours (review). Oncology Reports. 2009;21:1359-1366

[24] Rege TA, Wagner AJ, Corless CL, Heinrich MC, Hornick JL. “Pediatric-type” gastrointestinal stromal tumors in adults: Distinctive histology predicts genotype and clinical behavior. The American Journal of Surgical Pathology. 2011;35:495

[25] Pappo AS, Janeway KA. Pediatric gastrointestinal stromal tumors. Hematology/Oncology Clinics of North America. 2009;23:15-34

[26] Janeway KA, Kim SY, Lodish M, et al. Defects in succinate dehydrogenase in gastrointestinal stromal tumors lacking KIT and PDGFRA mutations. Proceedings of the National Academy of Sciences of the United States of America. 2011;108(1):314-318

[27] Killian JK, Kim SY, Miettinen M, et al. Succinate dehydrogenase mutation underlies global epigenomic divergence in gastrointestinal stromal tumor. Cancer Discovery. 2013;3(6):648-657

[28] Kindblom LG, Remotti HE, Aldenborg F, Meis-Kindblom JM. Gastrointestinal pacemaker cell tumor (GIPACT): Gastrointestinal stromal tumors show phenotypic characteristics of the interstitial cells of Cajal. The American Journal of Pathology. 1998;152: 1259-1269 
[29] Fletcher CD, Berman JJ, Corless C, et al. Diagnosis of gastrointestinal stromal tumors: A consensus approach. Human Pathology. 2002;33:459-465

[30] Sarlomo-Rikala M, Kovatich AJ, Barusevicius A, Miettinen M. CD117: A sensitive marker for gastrointestinal stromal tumors that is more specific than CD34. Modern Pathology. 1998;11:728-734

[31] Miettinen M, Lasota J. Gastrointestinal stromal tumors. Gastroenterology Clinics of North America. 2013;42:399-415

[32] Lee C-H, Liang C, Espinosa I. The utility of discovered on gastrointestinal stromal tumor 1 (DOG1) antibody in surgical pathology-the GIST of it. Advances in Anatomic Pathology. 2010;17:222-232

[33] Badalamenti G, Rodolico V, Fulfaro F, et al. Gastrointestinal stromal tumors (GISTs): Focus on histopathological diagnosis and biomolecular features. Annals of Oncology. 2007;18:vi136-vi140

[34] Miettinen M, Lasota J. Gastrointestinal stromal tumors: Pathology and prognosis at different sites. Seminars in Diagnostic Pathology. 2006;23:70-83

[35] Rubin BP, Heinrich MC, Corless CL. Gastrointestinal stromal tumour. The Lancet. 2007;369(9574):1731-1741

[36] Scherubl H, Faiss S, Knoefel WT, et al. Management of early asymptomatic gastrointestinal stromal tumors of the stomach. World Journal of Gastrointestinal Endoscopy. 2014;6(7):266-271

[37] Rossi S, Gasparotto D, Toffolatti L, et al. Molecular and clinicopathologic characterization of gastrointestinal stromal tumors (GISTs) of small size. The American Journal of Surgical Pathology. 2010;34:1480-1491

[38] Bennett JJ, Rubino MS. Gastrointestinal stromal tumors of the stomach. Surgical Oncology Clinics of North America. 2012;21:21-33

[39] Joensuu H, Hohenberger P, Corless CL. Gastrointestinal stromal tumour. Lancet. 2013;382: 973-983

[40] Gold JS, Dematteo RP. Combined surgical and molecular therapy: The gastrointestinal stromal tumor model. Annals of Surgery. 2006;244(2):176-184

[41] Dematteo RP, Maki RG, Antonescu C, et al. Targeted molecular therapy for cancer: The application of STI571 to gastrointestinal stromal tumor. Current Problems in Surgery. 2003;40(3):144-193

[42] Chaudhry UI, DeMatteo RP. Management of resectable gastrointestinal stromal tumor. Hematology/Oncology Clinics of North America. 2009;23:79-96 viii

[43] Wong CS, Gong N, Chu YC, et al. Correlation of measurements from diffusion weighted MR imaging and FDG PET/CT in GIST patients: ADC versus SUV. European Journal of Radiology. 2012;81:2122-2126 
[44] Corless CL, Barnett C, Heinrich M. Gastrointestinal stromal tumors: Origin and molecular oncology. Nature Reviews. Cancer. 2011;11:865-878

[45] Arne G, Nilsson B, Dalmo J, et al. Gastrointestinal stromal tumors (GISTs) express somatostatin receptors and bind radiolabeled somatostatin analogs. Acta Oncologica. 2013;52:783-792

[46] Chak A, Canto MI, Rösch T, et al. Endosonographic differentiation of benign and malignant stromal cell tumours. Gastrointestinal Endoscopy. 1997;45:468-473

[47] Vander Noot MR 3rd, Eloubeidi MA, Chen VK, et al. Diagnosis of gastrointestinal tract lesions by endoscopic ultrasound-guided fine-needle aspiration biopsy. Cancer. 2004;102:157-163

[48] DeWitt J, Emerson RE, Sharman S, et al. Endoscopic ultrasound-guided trucut biopsy of gastrointestinal mesenchymal tumor. Surgical Endoscopy. 2011;25:192-202

[49] Faigel DO, Abulhawa S. Gastrointestinal stromal tumors: The role of the gastroenterologist in diagnosis and risk stratification. Journal of Clinical Gastroenterology. 2012;46:629-636

[50] Tombesi P, Postorivo S, Catellani M, Tassinari D, Abbasciano V, Sartori S. Percutaneous ultrasonography guided core needle biopsy of gastrointestinal lesions: What's its actual role in clinical practice? A retrospective study for safety and effectiveness. Ultraschall in der Medizin. 2011;32(Suppl 1):S62-S67

[51] Miettinen M, El-Rifai W, HL Sobin L, et al. Evaluation of malignancy and prognosis of gastrointestinal stromal tumors: A review. Human Pathology. 2002;33(5):478-483

[52] Shiu MH, Farr GH, Papachristou DN, et al. Myosarcomas of the stomach: Natural history, prognostic factors, and management. Cancer. 1982;49(1):177-187

[53] Ng EH, Pollock RE, Munsell MF, et al. Prognostic factors influencing survival in gastrointestinal leiomyosarcomas. Implications for surgical management and staging. Annals of Surgery. 1992;215(1):68-77

[54] Rudolph P, Gloeckner K, Parwaresch R, et al. Immunophenotype, proliferation, DNA ploidy, and biological behavior of gastrointestinal stromal tumors: A multivariate clinicopathologic study. Human Pathology. 1998;29(8):791-800

[55] Goldblum JR, Appelman HD. Stromal tumors of the duodenum. A histologic and immunohistochemical study of 20 cases. The American Journal of Surgical Pathology. 1995;19(1):71-80

[56] Kiyabu MT, Bishop PC, Parker JW, et al. Smooth muscle tumors of the gastrointestinal tract. Flow cytometric quantitation of DNA and nuclear antigen content and correlation with histologic grade. The American Journal of Surgical Pathology. 1988;12(12):954-960

[57] Gunther T, Schneider-Stock R, Hackel C, et al. Telomerase activity and expression of hTRT and hTR in gastrointestinal stromal tumors in comparison with extragastrointestinal sarcomas. Clinical Cancer Research. 2000;6(5):1811-1818 
[58] DeMatteo RP. The GIST of targeted cancer therapy: A tumor (gastrointestinal stromal tumor), a mutated gene (c-kit), and a molecular inhibitor (STI571). Annals of Surgical Oncology. 2002;9(9):831-839

[59] Heinrich MC, Corless CL. Gastric GI stromal tumors (GISTs): The role of surgery in the era of targeted therapy. Journal of Surgical Oncology. 2005;90(3):195-207

[60] Kim MY, Jung HY, Choi KD, et al. Natural history of asymptomatic small gastric subepithelial tumors. Journal of Clinical Gastroenterology. 2011;45:330-336

[61] Agaimy A, Wunsch PH, Hofstaedter F, et al. Minute gastric sclerosing stromal tumors (GIST tumorlets) are common in adults and frequently show c-KIT mutations. The American Journal of Surgical Pathology. 2007;31(1):113-120

[62] Kawanowa K, Sakuma Y, Sakurai S. High incidence of microscopic gastrointestinal stromal tumors in the stomach. Human Pathology. 2006;37:1527-1535

[63] Roberts P, Eisenberg B. Clinical presentation of gastrointestinal stromal tumors and treatment of operable disease. European Journal of Cancer. 2002;38:S37-S38

[64] Choi SM, Kim MC, Jung GJ, et al. Laparoscopic wedge resection for gastric GIST: Long term follow-up results. European Journal of Surgical Oncology. 2007;33:444-447

[65] Hindmarsh A, Koo B, Lewis MP, et al. Laparoscopic resection of gastric gastrointestinal stromal tumors. Surgical Endoscopy. 2005;19:1109-1112

[66] Pierie JP, Choudry U, Muzikanski A, et al. The effect of surgery and grade on outcome of gastrointestinal stromal tumors. Archives of Surgery. 2001;136:383-389

[67] Novitsky YW, Kercher KW, Sing RF, et al. Long-term outcomes of laparoscopic resection of gastric gastrointestinal stromal tumors. Annals of Surgery. 2006;243(6):738-745

[68] Otani Y, Kitajima M. Laparoscopic surgery: Too soon to decide. Gastric Cancer. 2005;8: $135-136$

[69] Nakamori M, Iwahashi M, Nakamura M, et al. Laparoscopic resection for gastrointestinal stromal tumors of the stomach. American Journal of Surgery. 2008;196(3):425-429

[70] Nishimura J, Zheng ZC, Zhang JJ, et al. Surgical strategy for gastric gastrointestinal stromal tumors: Laparoscopic versus open resection. Surgical Endoscopy. 2007;21(6):875-878

[71] Otani Y, Furukawa T, Yoshida M, et al. Operative indications for relatively small $(2-5 \mathrm{~cm})$ gastrointestinal stromal tumor of the stomach based on analysis of 60 operated cases. Surgery. 2006;139(4):484-492

[72] Hiki N, Nunobe S, Matsuda T, Hirasawa T, Yamamoto Y, Yamaguchi T. Laparoscopic endoscopic cooperative surgery. Digestive Endoscopy. 2015;27:197-204

[73] Van der Zwan SM, De Matteo RP. Gastrointestinal stromal tumour: 5 years later. Cancer. 2005;104:1781-1788

[74] DeMatteo RP, Heinrich MC, El-Rifai WM, et al. Clinical management of gastrointestinal stromal tumors: Before and after STI-571. Human Pathology. 2002;33(5):466-477 
[75] DeMatteo RP, Owzar K, Antonescu CR, et al. Efficacy of adjuvant imatinib mesylate following complete resection of localized, primary gastrointestinal stromal tumor (GIST) at high risk of recurrence: the US intergroup phase II trial ACOSOG Z9000 (abstract). Data presented at the 2008 ASCO Gastrointestinal Cancers Symposium. Orlando (FL), January 25-27; 2008

[76] DeMatteo RP, Ballman KV, Antonescu CR, et al. Placebo-controlled randomized trial of adjuvant imatinib mesylate following the resection of localized, primary gastrointestinal stromal tumor (GIST). Lancet. 2009;373:1097-1104

[77] Choi H. Critical issues in response evaluation on computed tomography: Lessons from the gastrointestinal stromal tumour model. Current Oncology Reports. 2005;7(4):307-311

[78] Benjamin RS, Choi H, Macapinlac HA, et al. We should desist using RECIST, at least in GIST. Journal of Clinical Oncology. 2007;25:1760-1764

[79] Jager PL, Gieterma JA, van der Graaf WT. Imatinib mesylate for the treatment of gastrointestinal stromal tumours: Best monitored with FDG PET. Nuclear Medicine Communications. 2004;25:433-438

[80] Van den Abbeele AD, Badawi RD. Use of positron emission tomography in oncology and its potential role to asseses response to Imatinib mesylate therapy in gastrointestinal stromal tumours (GISTs). European Journal of Cancer. 2002;38(Suppl. 5):S60-S65

[81] Chen LL, Trent JC, Wu EF, et al. A missense mutation in KIT kinase domain I correlates with Imatinib resistance in gastrointestinal tumors. Cancer Research. 2004;64:5913-5919

[82] Tamborini E, Bonadiman L, Greco A, et al. A new mutation in KIT ATP pocket causes acquired resistance to Imatinib in a gastrointestinal stromal tumor patient. Gastroenterology. 2004;127:294-299

[83] Blay JY, Bonvalot S, Casali P, et al. Consensus meeting for the management of gastrointestinal stromal tumors: Report of the GIST consensus conference of 20-21 March 2004, under the auspices of ESMO. Annals of Oncology. 2005;16:566-578 